\title{
Performance Analyses of Optical Burst-Switching Networks
}

\author{
Zvi Rosberg, Hai Le Vu, Member, IEEE, Moshe Zukerman, Senior Member, IEEE, and \\ Jolyon White, Student Member, IEEE
}

\begin{abstract}
This paper provides a scalable framework for analysis and performance evaluation of optical burst-switching (OBS) networks. In particular, a new reduced load fixed point approximation model to evaluate blocking probabilities in OBS networks is introduced. The model is versatile enough to cover known OBS reservation policies such as just-enough-time, just-in-time, burst segmentation, and route-dependent priorities. The accuracy of the model is confirmed by simulation and the various policies are compared.
\end{abstract}

Index Terms-Optical burst-switching (OBS), Erlang fixed point approximation, reduced load, queueing model, loss probability.

\section{INTRODUCTION}

$\mathbf{R}$ ECENT technology advancements in optical packet switching (OPS) [3], [9], [13] give rise to the need for performance evaluation methodologies that will support business decisions on technology migration, network dimensioning, and service level agreements. Optical burst switching (OBS) [2], [7], [9], [11], [13], [14] has been proposed as a realistic OPS technique to exploit the terabit bandwidth of wavelength-division multiplexing (WDM) transmission technology.

In OBS, Internet protocol (IP) packets with a common destination arriving at the same ingress node are aggregated into large bursts, each being switched and routed individually. OBS can reduce switching time (processing and fabric reconfiguration) as only a single header and possibly a trailer are associated with each burst. In OBS, the header (also called label or control packet) precedes the burst payload and attempts to reserve the required switching and transmission resources at each switch and output link port along the route. The header can be transmitted either on the payload wavelength, or on separate

Manuscript received August 1, 2002; revised March 31, 2003. This paper was supported in part by the Australian Research Council. The work of J. White was supported in part by Redfern Photonics.

Z. Rosberg was with the ARC Special Research Center for Ultra-Broadband Information Networks, University of Melbourne, Melbourne, VIC 3010 Australia. He is now with the Department of Communication Systems Engineering, Ben Gurion University, Beer-Sheva 84105 Israel (e-mail: rosberg@ bgumail.bgu.ac.il).

H. L. Vu and J. White are with the ARC Special Research Center for Ultra-Broadband Information Networks, Department of Electrical and Electronic Engineering, The University of Melbourne, Melbourne, VIC 3010 Australia (e-mail: h.vu@ee.mu.oz.au).

M. Zukerman is with the ARC Special Research Center for Ultra-Broadband Information Networks, Department of Electrical and Electronic Engineering, The University of Melbourne, Melbourne, VIC 3010, Australia. He was visiting the Department of Electronic Engineering, City University of Hong Kong, Hong Kong SAR, China (November 2002 and July 2003) (e-mail m.zukerman@ee.mu.oz.au).

Digital Object Identifier 10.1109/JSAC.2003.815909 control wavelength(s). The payload follows the header without waiting for acknowledgment. At every switch, if the requested resources are available, the burst is transparently switched to its next hop; otherwise, the burst is blocked and a fraction or all of it is lost. Given the current technological gap between optical transmission rate and switching capacity, OBS aims to achieve end-to-end lightpath speed with high link utilization.

We assume that all switches support full wavelength conversion, whereby a burst can use any available wavelength at each link along its route. The cases of no and limited wavelength conversion [18] are currently under study and will be reported elsewhere.

Note that OBS differs from circuit switching in two main aspects. First, OBS bursts immediately follow their headers without waiting for a reservation acknowledgment. Since they cannot be buffered at the switching nodes, bursts may use bandwidth resources along several links and still be blocked and lost without completing their routes. In circuit switching, on the other hand, transmission starts only after an end-to-end path reservation is acknowledged. Second, in circuit switching, allocated resources are exclusively available to the end-to-end connection for its entire duration, while in OBS, the reserved resources at each switch and output link port are held only for the duration they are needed for switching and transmission of individual bursts. It is worth noting that in OBS networks, where link propagation delay is significantly larger than burst-transmission time, multiple bursts could simultaneously propagate not only the same route but also along the same link and wavelength [2].

OBS is also different from packet switching since OBS bursts are not buffered at the switches, while packets are. Moreover, due to reservations, OBS bursts use the path links in a timesynchronized manner, while packets use them asynchronously.

Several OBS reservation protocols have been proposed. In this paper, we consider the following proposals.

- A variation of the just-in-time (JIT) protocol [14], whereby the control packet is not aware of the burst length and reserves the relevant link bandwidth (if available) for the entire burst as soon as it arrives at the switch. We consider a variation without acknowledgment as the maximal burst size is relatively small compared with the link transmission capacity.

- The just-enough-time (JET) protocol [7], whereby the control packet is aware of the burst length and reserves the link bandwidth (if available) for the burst duration. The time between when the control packet makes a reservation and when the burst arrives is called the offset time. 
- Burst segmentation (BS) [1], where the control packet is aware of the burst length and reserves the wavelength (bandwidth) starting from the first moment it becomes available until the remainder of the burst is transmitted. The initial data within the burst is discarded until the required wavelength on the output fiber becomes free, whereupon the remainder of the burst is successfully transmitted. Another variant of BS is simulated in [11], where the tail of the served burst is dropped and the newly arrived packet starts immediately.

- Preemptive priority burst-service policies in each switching node, whereby the priority depends on the route a burst follows [16].

It is well accepted after almost two decades of extensive studies that exact solutions for a blocking network of the type presented above are unattainable. Even known product form solutions (e.g., for conventional circuit-switched network models) are NP-complete [5].

The difficulties related to network models have led OBS researchers to use a single-link model that may provide a crude approximation for the link blocking probability. The single node analyses have certain drawbacks since they do not consider network related issues such as the following two contrasting effects:

- the wastage of capacity caused by eventually unsuccessful bursts on the links they traverse before they are discarded;

- the reduced load resulting from blocking of bursts.

These drawbacks motivate a network modeling approach to investigate and compare blocking probabilities of various OBS reservation policies and to study the effect of burst routing, burst-admission control and other networking aspects. In this paper, we rely on the so-called reduced load fixed-point approximation whereby each link blocking probability is approximated by considering only the reduced offered load caused by the blocking on other links in the network.

The remainder of the paper is organized as follows. In Section II, we present in detail the reduced load fixed-point approximation. In Section III, we show how the approximation is applied to various OBS reservation policies such as JET, JIT, BS, and route-dependent priorities. In Section IV, we validate the approximation by simulation and in Section V, we compare the various policies by using our approximation method. Finally, the conclusions are presented in Section VI.

\section{REDUCED LOAD FiXed PoINT APPROXIMATION}

Consider a network with $J$ directional links labeled by $1,2, \ldots, J$, and suppose that link $j$ comprises $F_{j}$ optical fibers. Each optical fiber is used for transmission of data in one fixed direction only and serves up to $W$ concurrent logical channels using WDM. Let $N_{j}$ be the number of such channels, hereinafter wavelength channels, in link $j$ in a given direction. Thus, $N_{j}=W \cdot F_{j}$.

A fixed route $\boldsymbol{r}=\left(r_{1}, r_{2}, \ldots, r_{n}\right)$ of length $n$ (i.e., with $n$ hops) is an ordered set of links connecting a source node to a destination node. Let $\mathcal{R}$ be the set of all possible routes. In this paper, we assume fixed routing. However, we currently develop analytical tools to obtain the blocking probabilities under various adaptive routing schemes. We expect that adaptive routing will lead to significant reduction in blocking probabilities.

For first cut analytical results, we assume in this paper that bursts offered to route $\boldsymbol{r}$ arrive according to a Poisson process with rate $\lambda_{\boldsymbol{r}}$ and all arrival processes are independent. The Poisson burst arrival process may be justified considering the fact that in current WDM switches, the variance and the mean of the switch processing and configuration time is of the same order of magnitude [15] together with the fact that interaggregation time between two consecutive bursts tends to have a very low standard deviation to mean ratio. Thus, bursts may still be forwarded from the ingress node approximately according to a Poisson process. Note also that other burst arrival processes (under study) can be incorporated into our modular framework.

A burst offered to route $\boldsymbol{r}$ uses a single-wavelength channel from each link along the route until it is blocked or until it exits the network. That is, if the burst is first blocked by link $r_{j}$ it uses a single-wavelength channel from links $r_{1}, r_{2}, \ldots, r_{j-1}$ at subsequent time intervals shifted by the propagation delays of each subsequent link. We further assume that burst transmission times are independent and exponentially distributed and allow for different bandwidth capacities on the links. Let $\mu_{j}$ be the transmission rate of link $j$ measured in bursts per time unit.

A burst is in progress along a route $r$ at time $t$ if it uses at least one wavelength channel along that route at time $t$. Accordingly, a burst may be in progress even when some of the wavelength channels along its route are used by other bursts. Let $M$ be the product of $\max _{j} N_{j}$ and the maximum route length, and let $X_{\boldsymbol{r}}(t)$ be the number of bursts on route $r$ at time $t$. Also, let $Y_{\boldsymbol{r}}(t)$ be a vector of size $M$ whose $i$ th element (for $i \leq X_{\boldsymbol{r}}(t)$ ) is the time elapsed from the moment that the corresponding burst has finished its transmission on its assigned wavelength channel on the first link of its route (namely $r_{1}$ ) until time $t$. If the burst still uses a wavelength channel in $r_{1}$, then this element value is zero. All elements $i, i>X_{\boldsymbol{r}}(t)$, take the value zero.

Since the usage of subsequent wavelength channels by each burst are separated by fixed time intervals and the transmission times are exponential (memoryless), the joint process $\left\{Z_{r}(t)=\right.$ $\left.\left(X_{\boldsymbol{r}}(t), \boldsymbol{Y}_{\boldsymbol{r}}(t)\right) \mid \boldsymbol{r} \in \mathcal{R}, t \geq 0\right\}$ is a Markov process. Moreover, since $X_{\boldsymbol{r}}(t)$ takes a finite number of values and $\boldsymbol{Y}_{\boldsymbol{r}}(t)$ evolves linearly in time and varies within a bounded interval, the process has a stationary distribution.

Our aim is to evaluate the stationary blocking probability of an arbitrary burst and of a burst offered to route $\boldsymbol{r}$. Given the difficulty in obtaining exact mathematical results for this problem, we assume, as in [4], [10], and [17] that each blocking event occurs independently from link to link along any route.

Consider the process $\left\{Z_{\boldsymbol{r}}(t) \mid \boldsymbol{r} \in \mathcal{R}, t \geq 0\right\}$ under stationary conditions and let $\boldsymbol{B}=\left(B_{1}, B_{2}, \ldots, B_{J}\right)$ denote the vector of stationary link blocking probabilities. By the independence assumption above, it follows that the load offered to link $j, \rho_{j}$, satisfies:

$$
\rho_{j}=\mu_{j}^{-1} \sum_{\boldsymbol{r} \in \mathcal{R}: j \in \boldsymbol{r}} \lambda_{\boldsymbol{r}} \prod_{i=1}^{J}\left(1-I(i, j, \boldsymbol{r}) \cdot B_{i}\right)
$$


where $I(i, j, \boldsymbol{r})$ equals one or zero depending whether or not $i, j \in \boldsymbol{r}$ and link $i$ strictly precedes (not necessarily immediately) link $j$ along route $\boldsymbol{r}$, respectively.

Furthermore, the independence assumption implies that the offered load to each link $j$ is a Poisson process with rate $\rho_{j}$. Thus, the blocking probability is given by the following Erlang formula:

$$
B_{j} \stackrel{\text { def }}{=} E\left(\rho_{j}, N_{j}\right)=\frac{\rho_{j}^{N_{j}} / N_{j} !}{\sum_{k=0}^{N_{j}} \rho_{j}^{k} / k !} .
$$

Combining (1) and (2) yields the following Erlang fixed point (EFP) equations satisfied by the approximate link blocking probabilities:

$$
B_{j}=E\left(\mu_{j}^{-1} \sum_{\boldsymbol{r} \in \mathcal{R}: j \in \boldsymbol{r}} \lambda_{\boldsymbol{r}} \prod_{i=1}^{J}\left(1-I(i, j, \boldsymbol{r}) \cdot B_{i}\right), N_{j}\right) .
$$

Notice that the EFP equations impose consistency among the link blocking probabilities and their respective offered loads under the link independence assumption.

Resolving the vector $\boldsymbol{B}$ from the EFP (3) and invoking the independence assumption again, the approximate blocking probability of bursts offered to route $\boldsymbol{r}, B(\boldsymbol{r})$ satisfies

$$
B(\boldsymbol{r})=1-\prod_{i \in \boldsymbol{r}}\left(1-B_{i}\right)
$$

and the blocking probability of an arbitrary burst $B$, satisfies

$$
B=\frac{1}{\Lambda} \sum_{\boldsymbol{r} \in \mathcal{R}} \lambda_{\boldsymbol{r}} \cdot B(\boldsymbol{r})
$$

where $\Lambda=\sum_{\boldsymbol{r} \in \mathcal{R}} \lambda_{\boldsymbol{r}}$.

Suppose that burst lengths (in number of packets) are independent and identically distributed random variables. Then, assuming that the bursts are served based on the first-come firstserved (FCFS) scheduling order (e.g., in the case of zero offset times), the blocking probability of packets offered to route $\boldsymbol{r}$, and the blocking probability of an arbitrary packet are also given by $B(\boldsymbol{r})$ and $B$, respectively.

Interestingly, the OBS reduced load offered to link $j$ as given in (1) is larger than $\mu_{j}^{-1} \sum_{\boldsymbol{r} \in \mathcal{R}: j \in \boldsymbol{r}} \lambda_{\boldsymbol{r}} \prod_{i \in \boldsymbol{r} \backslash\{j\}}\left(1-B_{i}\right)$, the reduced load offered to link $j$ in a conventional circuit switching network.

The most efficient way to solve the link blocking probabilities (if a solution exists) is by the following successive substitution procedure. For any given vector of blocking probabilities $\boldsymbol{B}$, define the transformation vector $\boldsymbol{T}(\boldsymbol{B}) \stackrel{\text { def }}{=}\left(T_{1}(\boldsymbol{B}), \ldots, T_{J}(\boldsymbol{B})\right)$ by

$$
T_{j}(\boldsymbol{B})=E\left(\mu_{j}^{-1} \sum_{\boldsymbol{r} \in \mathcal{R}: j \in \boldsymbol{r}} \lambda_{\boldsymbol{r}} \prod_{i=1}^{J}\left(1-I(i, j, \boldsymbol{r}) \cdot B_{i}\right), N_{j}\right) .
$$

We begin the successive substitution procedure with a certain initial blocking probability vector $\boldsymbol{B}$ and repeatedly apply the transformation $\boldsymbol{T}(\boldsymbol{B})$. That is, we compute $\boldsymbol{B}^{n}=\boldsymbol{T}\left(\boldsymbol{B}^{n-1}\right)$ for $n=1,2, \ldots,\left(\right.$ where $\left.\boldsymbol{B}^{0}=\boldsymbol{B}\right)$, until $\boldsymbol{B}^{n}$ is sufficiently close to $\boldsymbol{B}^{n-1}$
Observe that the transformation $\boldsymbol{T}(B)$ is a continuous mapping from the compact set $[0,1]^{J}$ to itself and, therefore, it has a fixed point by the Brouwer fixed-point theorem [6]. We cannot establish the uniqueness of the solutions as has been established in [4] for the conventional circuit switching network. Nevertheless, in all the numerical examples used for this study, the iterations always converged to a unique fixed point regardless of the initial vector. The following convergence properties can be established.

Since the transformation $\boldsymbol{T}(\boldsymbol{B})$ is decreasing, namely, $\boldsymbol{T}(\boldsymbol{B})<\boldsymbol{T}\left(\boldsymbol{B}^{\prime}\right)$ whenever $\boldsymbol{B}>\boldsymbol{B}^{\prime}$, the following result is easily derived (as done in [17] for the circuit switching network).

Theorem 1: Starting with $B^{0}=1$ (the vector of all ones), then the successive substitution for every $n$ yields the following upper and lower bounds on all fixed-point solutions $B^{\star}=T\left(B^{\star}\right)$

$$
\hat{\boldsymbol{B}}=\boldsymbol{B}^{1}<\boldsymbol{B}^{2 n+1}<\boldsymbol{B}^{2 n+3}<\boldsymbol{B}^{\star}
$$

and

$$
B^{\star}<B^{2 n+2}<B^{2 n}<B^{0}=1
$$

where $\hat{\boldsymbol{B}}=\left(\hat{B}_{1}, \hat{B}_{2}, \ldots, \hat{B}_{J}\right)$ and $\hat{B}_{j}$ is the total external arrival rate to link $j$. Furthermore, the sequence $\left\{B^{2 n}\right\}$ converges to an upper bound $\overline{\boldsymbol{B}}$ and the sequence $\left\{\boldsymbol{B}^{2 n+1}\right\}$ converges to a lower bound $\underline{B}$ on every fixed-point solution.

Since the transformation $T(B)$ is decreasing, $\underline{B}=\boldsymbol{T}(\bar{B})$ and $\bar{B}=T(\underline{B})$. Therefore, if the lower and the upper bounds are not sufficiently close, one may restart the iterations from a new value $B^{0}$ such that $\underline{B}<B^{0}<\bar{B}$ and $B^{1}, B^{2}<B^{0}$ (alternatively, $\boldsymbol{B}^{1}, \boldsymbol{B}^{2}>\boldsymbol{B}^{0}$ ). Since the transformation $\boldsymbol{T}(\boldsymbol{B})$ is decreasing, it will shrink the gap between the previous bounds and eventually will drive the iterations to a fixed-point solution. If there is a unique fixed point, it will eventually find it. As mentioned above, in all the examples used for this study, the iterations always converge. Moreover, since the upper and lower bounds were the same, the converging value is the unique fixedpoint solution.

Due to the hierarchical nature of our solution, the Erlang formula in the EFP (3) can be replaced by other blocking probabilities as explained in Section III. For such cases, convergence of the odd and even subsequences of the iterations to the lower and upper bounds, respectively, is also guaranteed as long as the transformation $\boldsymbol{T}(\boldsymbol{B})$ is decreasing.

\section{EXTENSIONS AND APPLICATIONS}

As discussed above, the EFP equations and the successive substitution iterations can be applied to JIT and JET, as well as extended to other policies. Below, we show how to apply them to JET and JIT and derive the equations for policies that alow burst preemption and BS.

\section{A. JET and JIT Policies}

The formulae obtained in Section II can be applied to the JIT and the JET regimes. With JET, the wavelength is reserved from the moment the burst will actually arrive. Therefore, the 
transmission rate $\mu_{j}$ at link $j$ is set to $1 / E\left(S_{j}\right)$, where $S_{j}$ is the actual burst service time in link $j$. With JIT, the wavelength is reserved as soon as the control packet arrives at the switch. Thus, we set $\mu_{j}=1 /\left(E\left(S_{j}\right)+E\left(D_{j}\right)\right)$, where $D_{j}$ is the offset time between the control packet and its corresponding burst arrivals to the switching node of link $j$. Otherwise, (1)-(3) are left intact.

Note that with JET and JIT, serviced bursts are never preempted. Furthermore, in our model we assume that burst-transmission time is exponential with a link dependent mean, but the mean burst-transmission times, on different wavelengths of the same link are identical. The exponential transmission times give rise to interesting blocking probability issues. Assuming offset times are independent of their corresponding burst sizes, different offset times for different bursts do not affect burst blocking probability. The reason for this is that bursts can be swapped and, due to the exponential assumption, the mean forward recurrence time of one burst is equal to the mean service time of the other [12]. However, different offset times can cause a burst to be blocked by another burst that arrives later. In such a case, when the blocked burst arrives, there is work to be done in the system while the system is not serving. That is, the system is not work conserving. This leads to higher workload loss and, therefore, higher packet blocking probability. This is consistent with the previous argument as the later burst that its transmission time is equal to the forward recurrence time of the earlier burst is on average shorter than the earlier burst. In other words, the non-FCFS scheduling will, on average, discard longer bursts in favor of shorter bursts. If burst-transmission time is nonexponential, the offset times are likely to affect both burst-blocking probability and packet blocking probability. In any case, non-FCFS scheduling is less efficient than FCFS scheduling and, thus, leads to higher packet blocking probability. In this paper, we assume that the bursts are served FCFS.

\section{B. Burst Segmentation (BS)}

Since an OBS burst is an aggregation of many IP packets, there have been proposals to reduce packet loss probability by dumping a part of one of the two colliding bursts only during the collision time [1], [11]. For such proposals, we use the generic name burst segmentation. We consider the variant solved analytically in [1] [called there optical composite burst switching (OCBS)], where only the initial part of the new arriving burst is discarded until a wavelength becomes free on the output fiber. From that instant, the remainder of the burst is successfully transmitted. Note that wavelength scheduling is immaterial in BS since every wavelength that becomes available serves a fragment of one of the waiting bursts.

We now introduce a simple analytical model to approximate the blocking probability of a single IP packet (rather than of a burst - since bursts can be fragmented and partially served) in a single isolated link under burst Poisson arrivals. Our approximation is asymptotically exact as the number of packets per burst approaches infinity. To evaluate the packet loss probability in the case of a network, this approximation can replace the Erlang formula in (2). Note also that an exact solution for IP packet loss probability under the assumption that packets are exponentially distributed appears in [1]. In principle, the solution of [1] can also replace (2).
The underlying idea in our approach is to model the link by an $M / G / \infty$ queue (rather than by an $M / G / N / N$ queue). We will show that there is a one-to-one mapping between the states of the $M / G / \infty$ system and that of the burst-segmentation system. Let $N$ be the number of available wavelength channels in the single link under consideration, and let $A$ be the traffic load offered to the $M / G / \infty$ system. The load $A$ equals the traffic load offered to the link under consideration.

The state of the $M / G / \infty$ queue is the number of busy servers denoted $i$. Consider two cases of the state: 1) $0 \leq i \leq N$ and 2) $i>N$. In case 1 ), $i$ in the $M / G / \infty$ system is equivalent to the state of having $i$ wavelength channels busy in the burstsegmentation system. In case 2), $i$ in the $M / G / \infty$ system is equivalent to the state of having $N$ wavelength channels busy in the burst-segmentation system and $i-N$ additional bursts that are being dumped. That is, if $i=N+k$, then $k$ bursts are losing bits (not necessarily integral parts of packets), as there is no means to buffer the packets in optical switching. However, once one of the $N$ servers (wavelength channels) becomes free, the remainder of that burst is allocated to the free wavelength channel and immediately starts service.

Let $P(i)$ be the stationary probability that $i$ servers are busy in the $M / G / \infty$ system above. It is well known that the number of busy servers in an $M / G / \infty$ model has a Poisson distribution with parameter $A$. Thus, $P(i)=A^{i}\left(e^{-A} / i !\right), i=1,2, \ldots$.

By definition, the stationary probability of a state is the long-run proportion of time that the system stays at that state. Thus, when the system is in state $i, i>N$, the workload loss rate is $i-N$ and the mean loss rate, denoted by $E[L]$, is, therefore

$$
E[L]=1 \cdot P(N+1)+2 \cdot P(N+2)+3 \cdot P(N+3)+\cdots .
$$

The fraction of the workload lost out of the total offered workload is

$$
B=\frac{E[L]}{A} .
$$

Notice that in the above analysis, we are not considering the following additional loss. Assume we are in case 2) above, with $N>i$, and consider a point in time, denoted $t_{f}$, in which a wavelength channel becomes free. At $t_{f}$, one of the bursts that was being dumped until $t_{f}$, is being served by the free channel from $t_{f}$ onwards. It is most likely that $t_{f}$ will not coincide exactly with packet boundary. Therefore, the remainder of the packet, even if it is transmitted by the free channel, will be lost. In other words, if a fraction of a packet is lost, the entire packet must be lost. In this sense, our approximation is optimistic. However, the error is in the order of half a packet per the number of packets comprising a burst. This ratio approaches zero as the number of packets per bursts approaches infinity.

Thus, when the ratio between average packet size and average burst size is very small (which is the typical case), (5) is expected to provide a good approximation. We observed that our approximation matches the exact solution of [1] for the parameter range provided there. To find the blocking probabilities in an OBS network with $\mathrm{BS}$, we replace (2) for every link $j$ by (5), where $A$ assumes the value of the reduced offered workload $\rho_{j}$. This is valid since the blocking probability in every link is a function 
of the workload, regardless of the burst and packet length distributions.

\section{Route-Dependent Priorities}

Preemptive priority burst-service policies in each switching node that depend on the burst route [16] can reduce the overall blocking probability. It can also achieve other performance measures based on, e.g., routes and fairness. Here, we show how to evaluate the burst-blocking probabilities when such policies are used.

For any route $\boldsymbol{r}$ and link $j$, let $h(\boldsymbol{r})$ be the number of hops of route $\boldsymbol{r}$, and $t(\boldsymbol{r}, j)$ be the number of hops of route $r$ until and including link $j$. A route-dependent service priority at link $j$ could be any one-to-one mapping from the set of pairs $\{(h(\boldsymbol{r}), t(\boldsymbol{r}, j))\}$ into an ordered set of priority indices. The lower the priority index, the higher its service priority. Since we consider only fixed routing policies (that do not depend on the network state), any such route-dependent policy determines a static preemptive priority service policy at each link. Note that a given burst may be mapped into a different priority in each link along its route.

As for JET and JIT, we assume FCFS scheduling order, within each priority class, for our route-dependent preemptive regimes. Note, however, that some priority side-effects related to offset times can be taken into consideration by our framework. For example, by giving higher priority to bursts that still have many hops ahead of them (i.e., long offset time) versus bursts that have fewer hops to go (shorter offset time). This offset time consequence may lead to inefficiencies, so the network designer may need to compensate by increasing priority to those bursts near the end of their paths as we do in Section IV. We, however, must keep in mind that further disrupting the FCFS policy leads to inefficiencies.

For a given isolated link where the offered loads of bursts from priority classes $p$ arrive as independent Poisson processes with rates $\rho^{p}, p \in \mathcal{P}=\{1,2, \ldots, P\}$, the burst-blocking probabilities have been derived in [12]. Denote by $B_{j}^{p}$ the blocking probability of a priority $p$ burst in link $j$, and by $p(r, j)$ the priority of a route $\boldsymbol{r}$ burst in link $j$. These blocking probabilities are expressed only as function of $\rho^{p}, p \in \mathcal{P}$.

Under the blocking independence assumption above it follows that the reduced load of route $\boldsymbol{r}$ bursts offered to link $j, \rho_{j}(\boldsymbol{r})$, satisfies

$$
\rho_{j}(\boldsymbol{r})=\mu_{j}^{-1} \lambda_{\boldsymbol{r}} \prod_{i=1}^{J}\left(1-I(i, j, \boldsymbol{r}) \cdot B_{i}^{p(\boldsymbol{r}, i)}\right) .
$$

For each link $j$, let $\rho_{j}^{p}$ be the reduced load of bursts from priority $p$ arriving to link $j$. Observe that the set of reduced loads $\left\{\rho_{j}(\boldsymbol{r}), p(\boldsymbol{r}, j) \mid \boldsymbol{r} \in \mathcal{R}\right\}$ arriving to link $j$, determines the set $\left\{\rho_{j}^{p} \mid p \in \mathcal{P}\right\}$. Therefore, the set of new blocking probabilities $\left\{B_{j}^{p} \mid p \in \mathcal{P}\right\}$ can be computed from the reduced loads. The successive substitution iterations for these priority policies becomes similar to the case without priorities (derived in Section II) after replacing each $\rho_{j}$ and $B_{j}$ with the vectors $\boldsymbol{\rho}_{j}=$ $\left(\rho_{j}^{1}, \ldots, \rho_{j}^{P}\right)$ and $B_{j}=\left(B_{j}^{1}, \ldots, B_{j}^{P}\right)$, respectively.

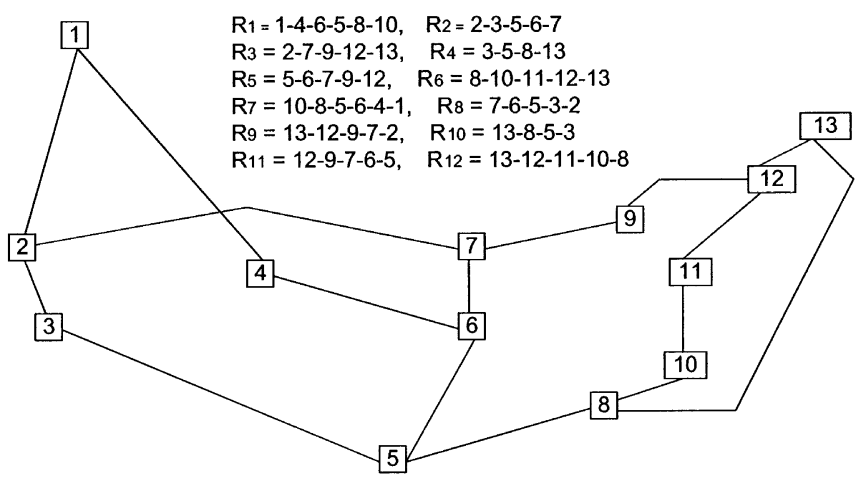

Fig. 1. NSFNET backbone network (T3, 45 Mb/s, April 1995) and our experimental routes.

\section{Fixed Point ApPROXIMATION VALIDATION}

We use the NSFNET backbone network topology ${ }^{1}$ depicted in Fig. 1 as a test bed to verify the independence assumption (used for our reduced load fixed-point approximation) against results from a Markov process simulation. The network topology comprises 13 OBS switches labeled $1,2, \ldots, 13$, and 32 unidirectional fiber links, each comprising $W=8$ wavelength channels. For this network we evaluate the blocking probabilities of 12 routes defined in Fig. 1. The selected routes represent a variety of path lengths, link sharing degrees and mixtures of external and on-route internal traffic processes. All routes are shortest paths, except for $R_{3}$ and $R_{7}$ selected to obtain better route diversity.

For the verification process, we consider symmetric external arrival streams, where each route is offered the same external rate of bursts. To represent a network with various loads we consider a set of different arrival rates of $\{30,50,80,100,120,150$, $180\}$ bursts/s for each route. The wavelength channel capacity and the burst length are set to yield a wavelength channel service rate $\mu$ of 25 bursts/s.

Our objective is to validate our approximation against simulation for JET, BS, and the following route-dependent priority policy, referred to as least remaining hop-count first (LRHF). With LRHF, in every wavelength channel, each transmitted burst is preempted by any newly arrived burst that has a strictly less remaining number of hops to its destination. Another priority policy is also evaluated in Section V. The approximation and the simulation results for blocking probability values as low as $10^{-5}$ are presented in Figs. 2-6. Due to space limitations, we cannot present the results for all routes, but the approximation quality is similar.

In Figs. 2 and 3, we show the blocking probabilities of JET for routes 1-4 and 9-12; in Fig. 4, we show the blocking probabilities of BS for routes 5-8; and in Fig. 5, we show the blocking probabilities of LRHF. All probabilities in these figures are derived using both approximation and simulation techniques. The simulation results are presented with their respective $95 \%$ confidence intervals based on student's t-distribution. (Note that the 95\% confidence intervals are so small that at times, it is hard to see them in the figures.)

\footnotetext{
${ }^{1}$ http://moat.nlanr.net/INFRA/NSFNET.html
} 

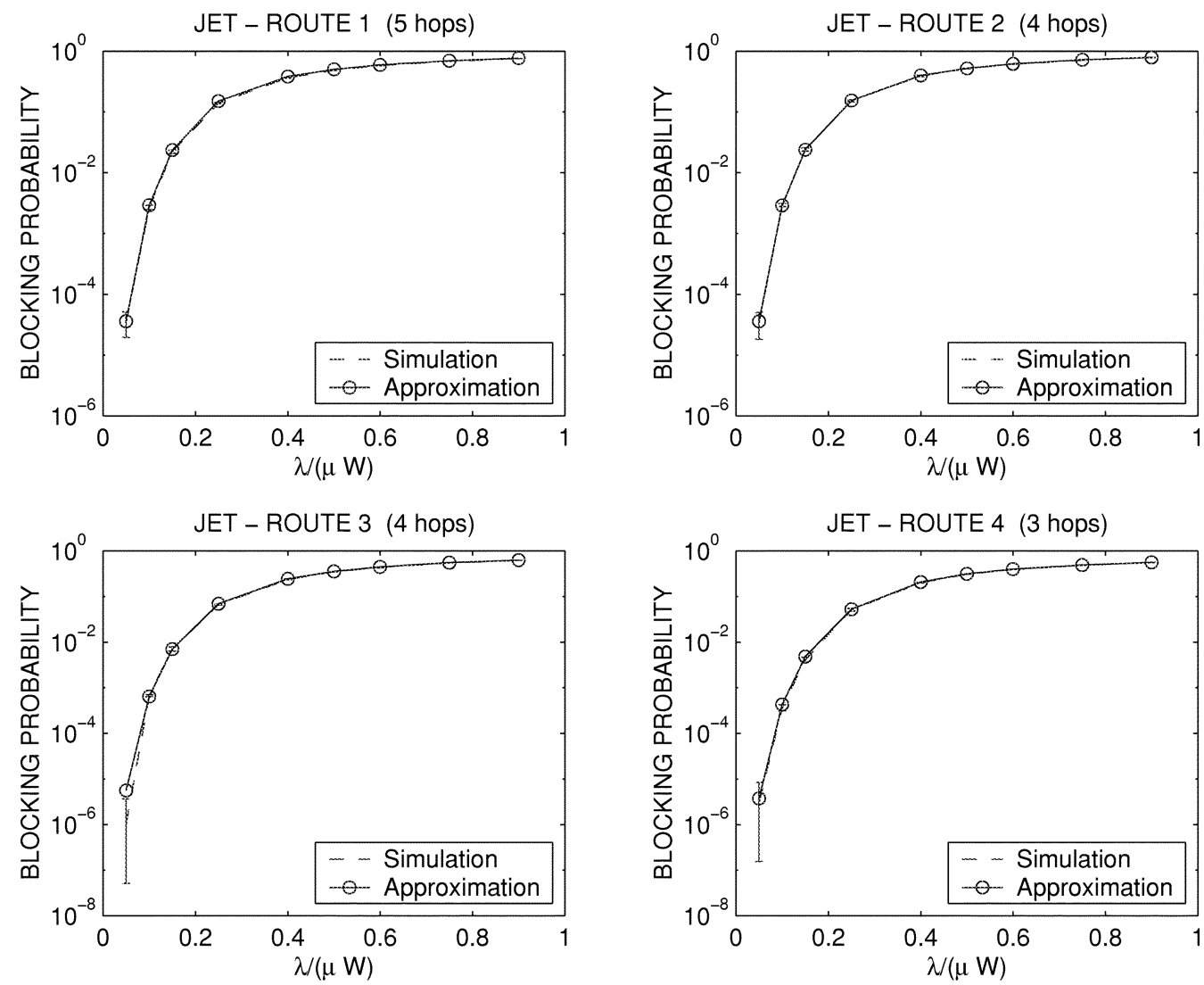

Fig. 2. Route blocking probabilities (approximation versus simulation) using JET.
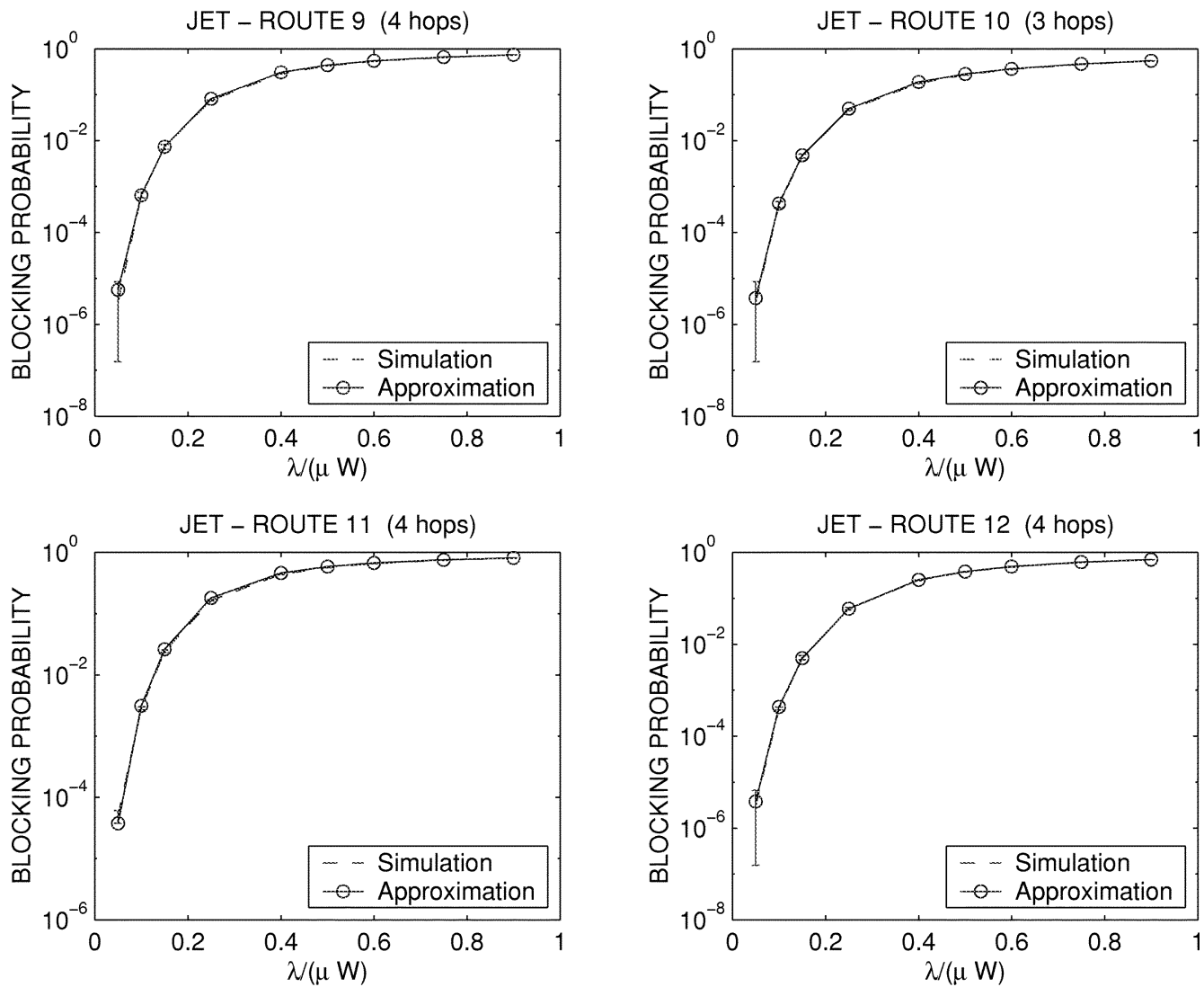

Fig. 3. Route blocking probabilities (approximation versus simulation) using JET. 

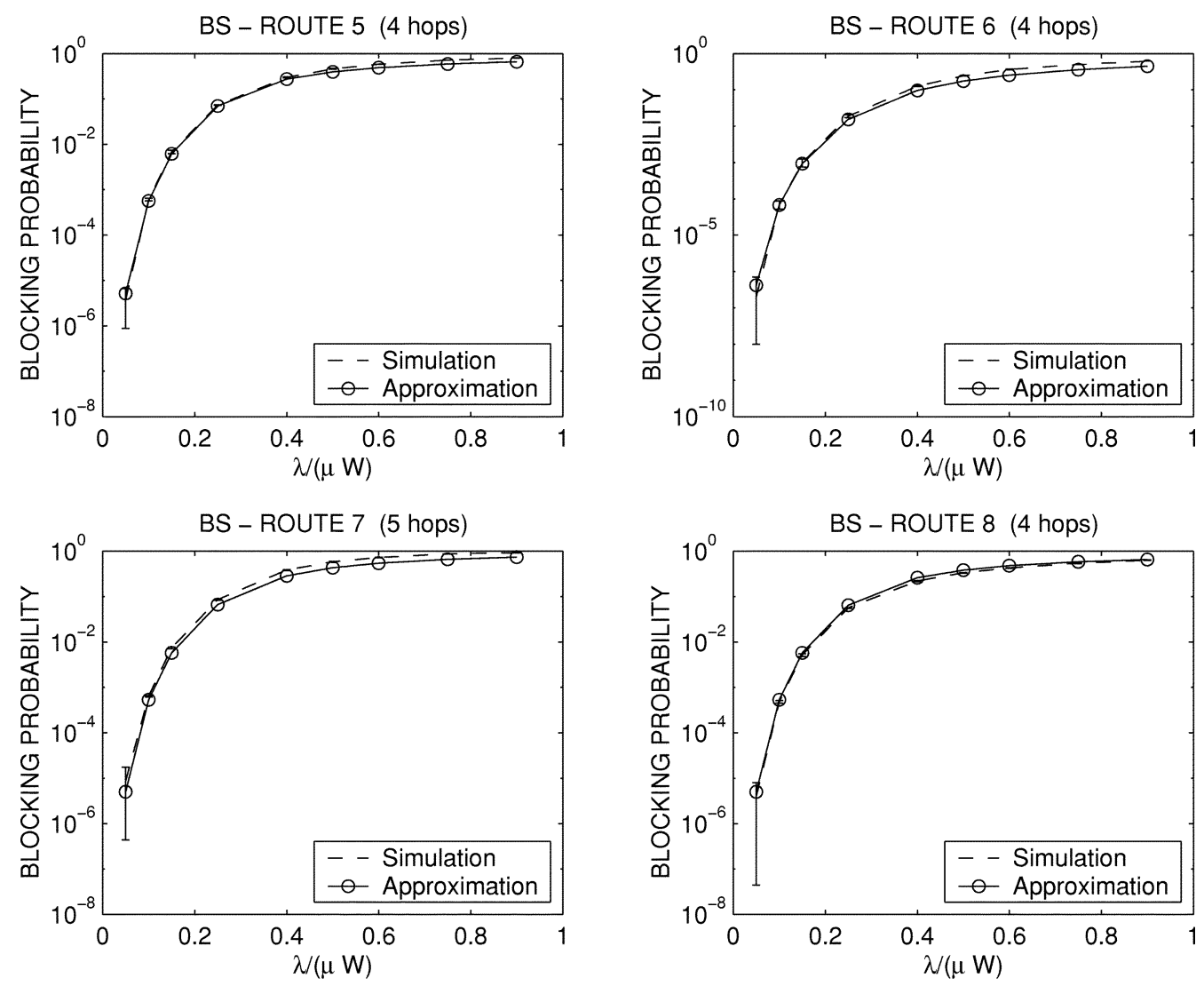

Fig. 4. Route blocking probabilities (approximation versus simulation) using BS.
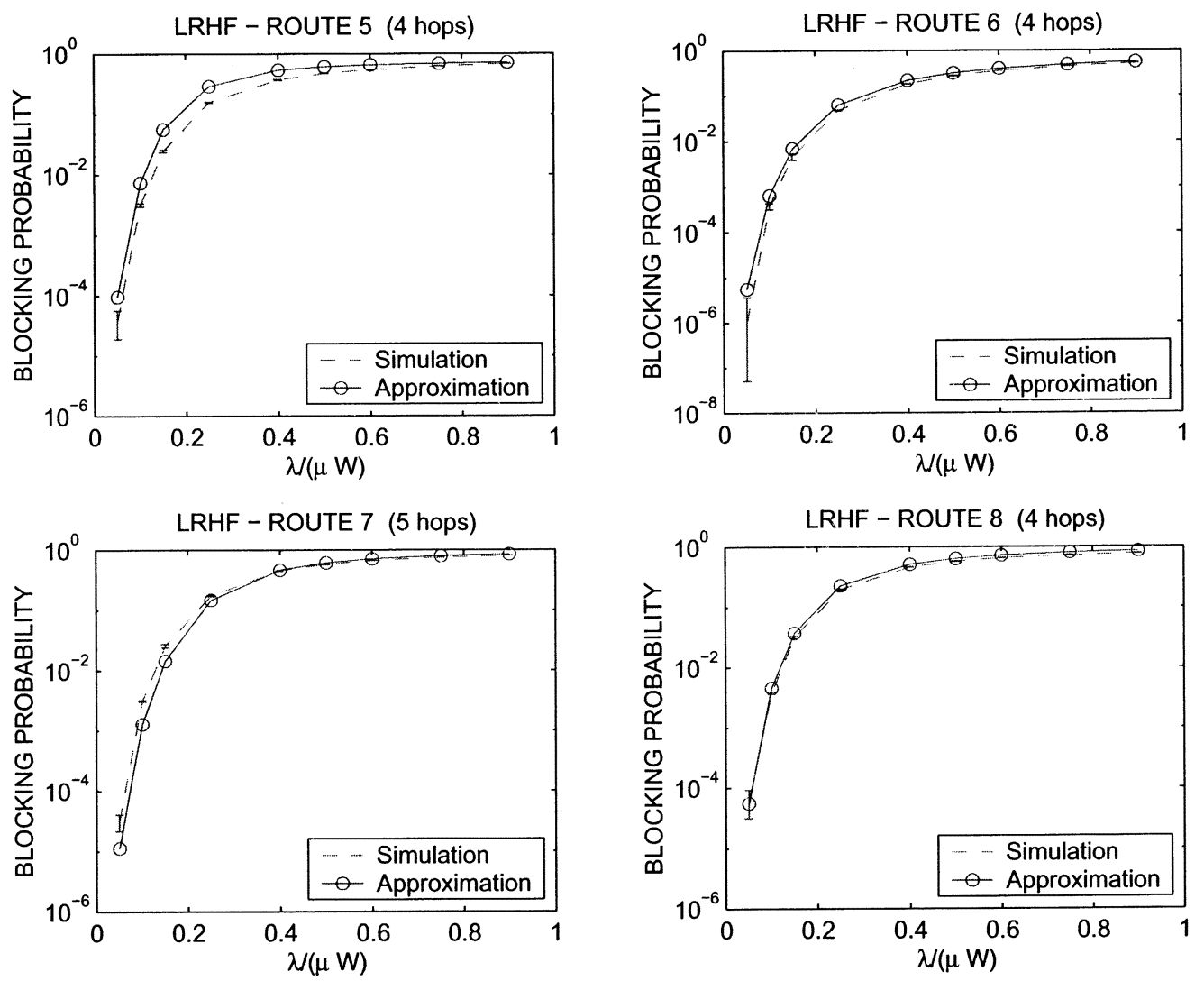

Fig. 5. Route blocking probabilities (approximation versus simulation) using LRHF. 
TABLE I

BLOCKING PROBABILITIES FOR VARIOUS LOADS-APPROXIMATION AND SIMULATION

\begin{tabular}{c||c|c||c|c}
\hline \multicolumn{1}{l|}{ Route (Policy) $\backslash$ Arrival Rate } & \multicolumn{2}{c||}{30} & \multicolumn{2}{c}{50} \\
\hline & Approx. & Sim. & Approx. & Sim. \\
\hline$R_{1}$ (JET) & 0.0237 & $0.0222 \pm 0.0013$ & 0.1512 & $0.1404 \pm 0.0023$ \\
\hline$R_{2}$ (JET) & 0.0238 & $0.0240 \pm 0.0016$ & 0.1545 & $0.1529 \pm 0.0057$ \\
\hline$R_{3}$ (JET) & 0.0071 & $0.0071 \pm 0.0008$ & 0.0692 & $0.0668 \pm 0.0015$ \\
\hline$R_{4}$ (JET) & 0.0048 & $0.0041 \pm 0.0005$ & 0.0525 & $0.0514 \pm 0.0034$ \\
\hline$R_{5}(\mathrm{JET})$ & 0.0258 & $0.0259 \pm 0.0019$ & 0.1650 & $0.1618 \pm 0.0034$ \\
\hline$R_{6}$ (JET) & 0.0048 & $0.0049 \pm 0.0007$ & 0.0499 & $0.0481 \pm 0.0025$ \\
\hline$R_{7}(\mathrm{JET})$ & 0.0240 & $0.0241 \pm 0.0013$ & 0.1627 & $0.1612 \pm 0.0048$ \\
\hline$R_{8}(\mathrm{JET})$ & 0.0238 & $0.0221 \pm 0.0017$ & 0.1528 & $0.1424 \pm 0.0014$ \\
\hline$R_{9}$ (JET) & 0.0074 & $0.0074 \pm 0.0009$ & 0.0824 & $0.0761 \pm 0.0027$ \\
\hline$R_{10}(\mathrm{JET})$ & 0.0048 & $0.0046 \pm 0.0005$ & 0.0500 & $0.0468 \pm 0.0020$ \\
\hline$R_{11}$ (JET) & 0.0262 & $0.0243 \pm 0.0010$ & 0.1802 & $0.1647 \pm 0.0033$ \\
\hline$R_{12}(\mathrm{JET})$ & 0.0050 & $0.0051 \pm 0.0005$ & 0.0594 & $0.0592 \pm 0.0034$ \\
\hline Overall (JET) & 0.0151 & $0.0147 \pm 0.00033$ & 0.1108 & $0.1060 \pm 0.00094$ \\
\hline Overall (LRHF) & 0.0149 & $0.0145 \pm 0.00029$ & 0.1024 & $0.1050 \pm 0.00090$ \\
\hline Overall (BS) & 0.0035 & $0.0034 \pm 0.00013$ & 0.0427 & $0.0418 \pm 0.00071$ \\
\hline
\end{tabular}

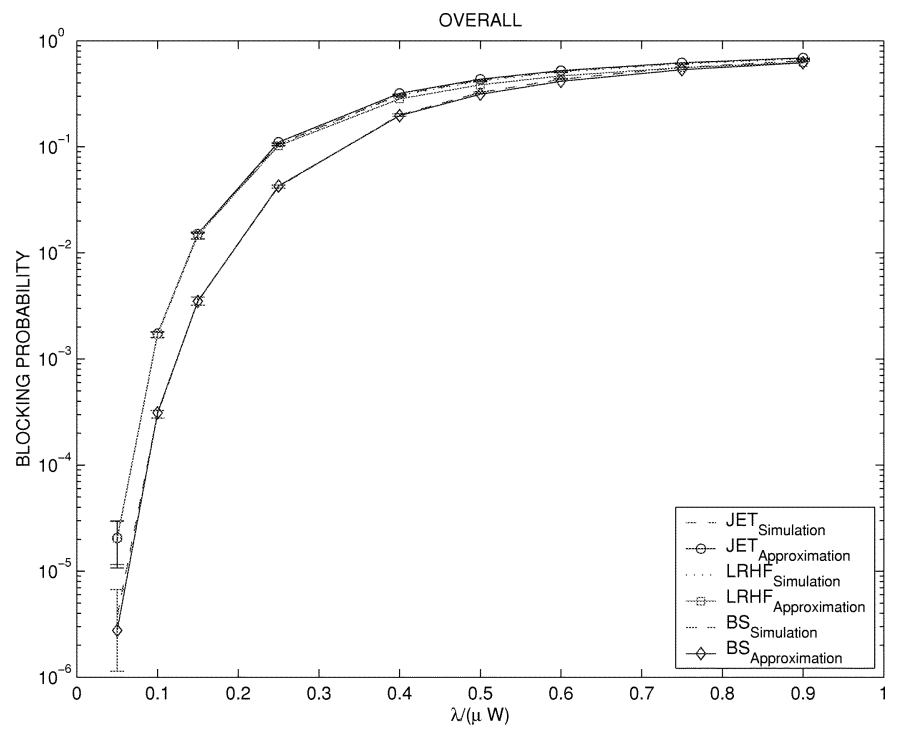

Fig. 6. Overall blocking probabilities (approximation versus simulation) using JET, BS, and LRHF.

The first 12 rows of Table I show the precise values of the approximation and simulation confidence intervals for the blocking probability of all routes using JET. We observe that the simulation results for the overall blocking probabilities agree very well with the approximation for all policies. A similar agreement can be observed for route blocking using the JET and BS policies. For the LRHF policy, there is a deviation for some routes but for most of them the agreement is very good.

Fig. 6, validates the approximation for the overall blocking probability using different low, medium, and high traffic loads for JET, for the LRHF priority, and for BS. The last three rows of Table I show the precise values of some of the results presented in Fig. 6. Again, the simulation results are presented with their respective $95 \%$ confidence intervals based on student's t-distribution. The results are again consistent.

The validation is performed for blocking probability values as low as $10^{-5}$ and in the next section, we will use our analytical approximation to compare the various OBS alternatives and policies.

\section{PERFORMANCE COMPARISON}

As explained above, and recalling our FCFS assumption, the burst-blocking probability for any policy except for BS is equal to its corresponding packet blocking probability. Therefore, to use a common scale, all probabilities below can be interpreted as packet blocking probabilities.

In Figs. 7-9, we present the route and overall blocking probabilities obtained by our approximation method under JET, BS, LRHF, and the following most traversed hop-count first (MTHF) priority scheme. With MTHF, bursts that have traversed the largest number of hops have the highest priority. This policy intends to protect bursts that have already used up significant network resources; whereas LRHF intends to protect bursts that are expected to use less network resources.

From Figs. 7-9, one may observe the following.

- From Fig. 9, BS has the lowest overall blocking probability, while the others achieve similar values. Furthermore, in the range between low to medium loads, BS is significantly better than the other policies.

- From Fig. 9, both priority policies improve the overall blocking probability of JET, but not significantly. Between the two priority policies, LRHF performs better.

- As expected, the MTHF policy improves the blocking probabilities of long routes provided that their prefixes do not collide with higher or equal priority routes (see ROUTE 1 in Fig. 7). If their prefixes do collide with higher or equal priority routes, then it still improves their blocking probabilities, but less than the improvement provided by BS (see ROUTE 7 in Fig. 8).

- As expected, the LRHF policy has an effect similar to that of the MTHF policy, but on short routes. That is, the blocking probabilities of short routes are reduced provided that their suffixes do not collide with higher or equal priority routes (see ROUTE 10 in 8). If their suffixes do collide with higher or equal priority routes, then it still improves their blocking probabilities, but less than the improvement provided by BS (see ROUTE 4 in Fig. 7). 

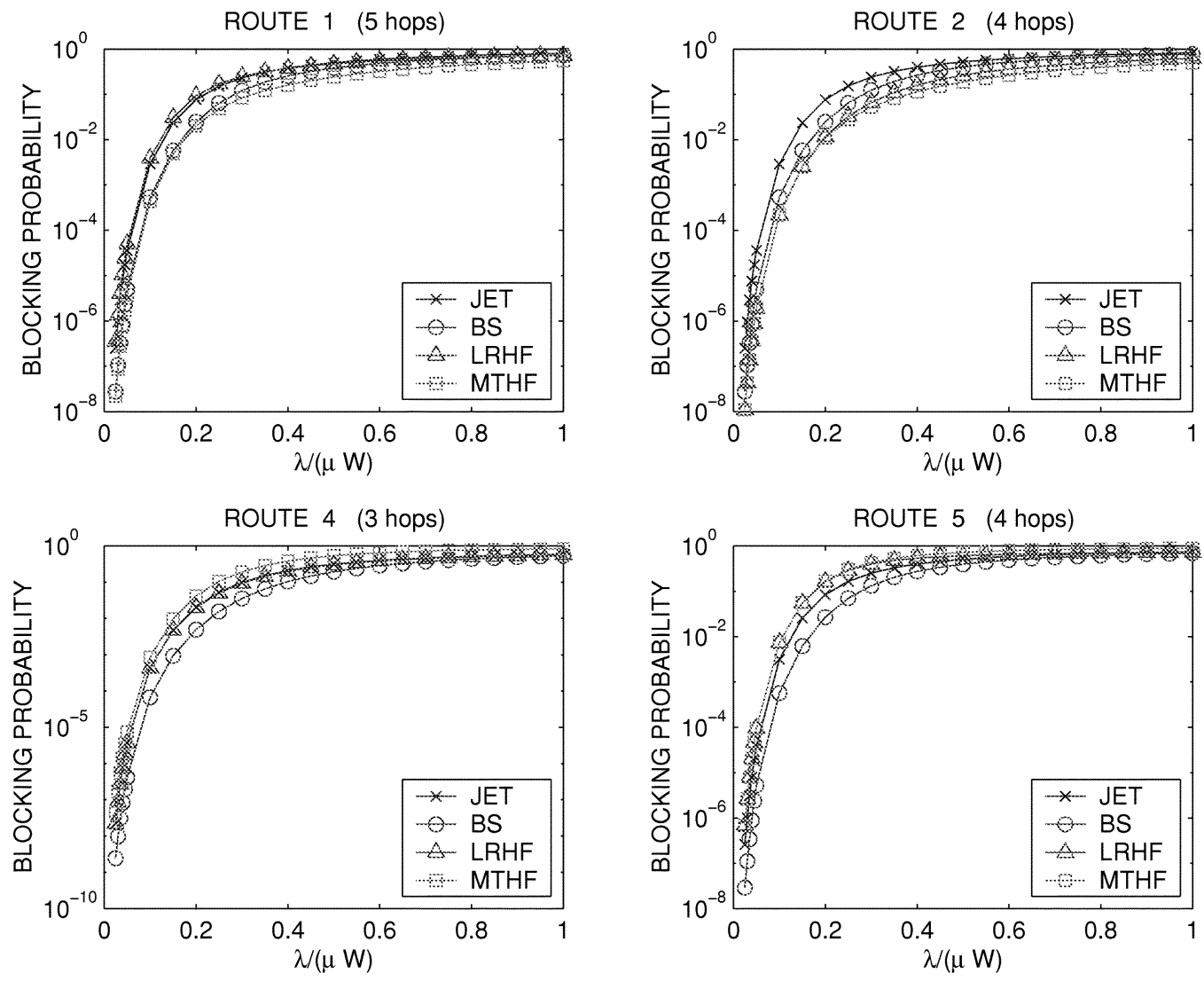

Fig. 7. Route blocking probabilities (approximation) using JET, BS, LRHF, and MTHF policies.
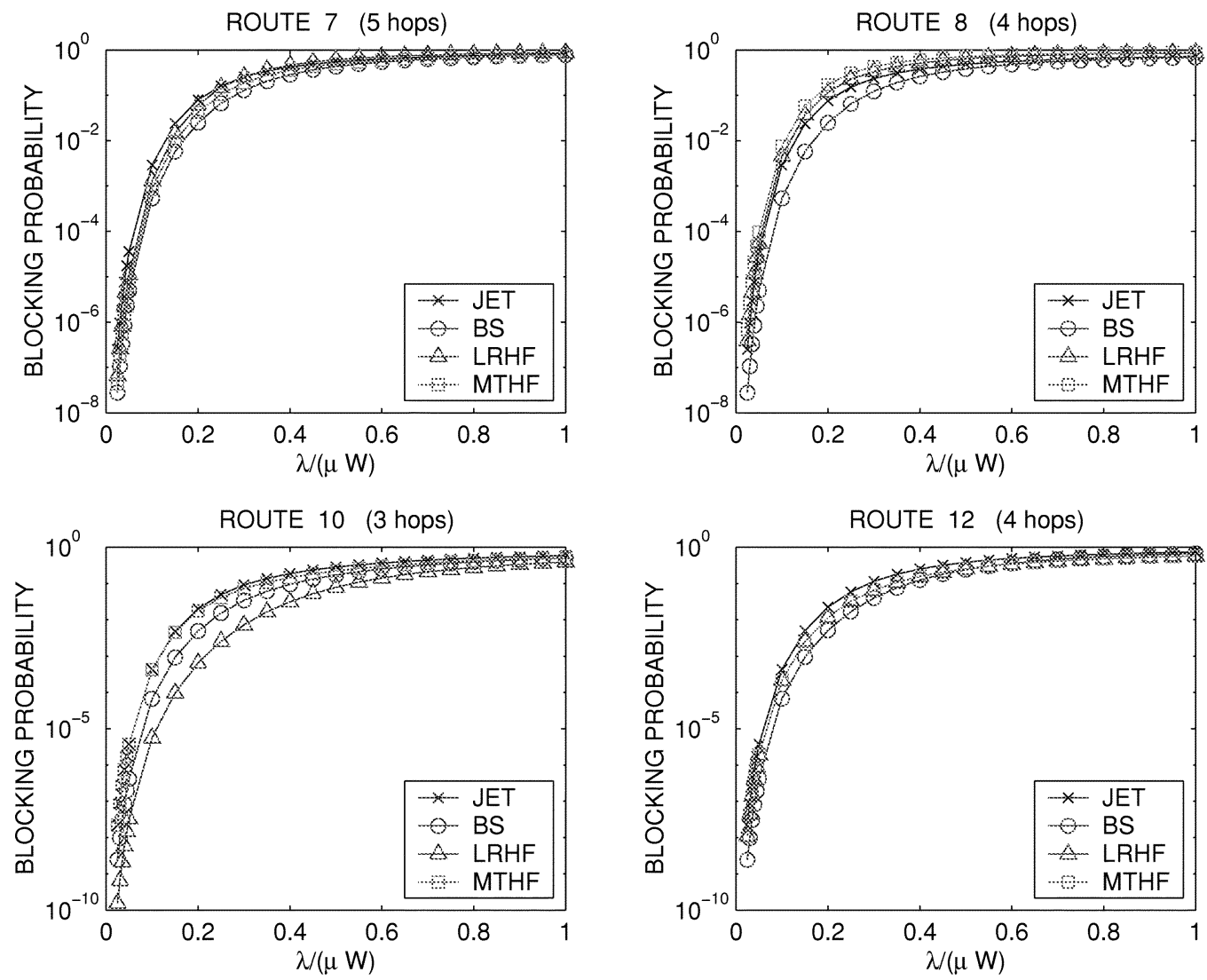

Fig. 8. Route blocking probabilities (approximation) using JET, BS, LRHF, and MTHF policies. 


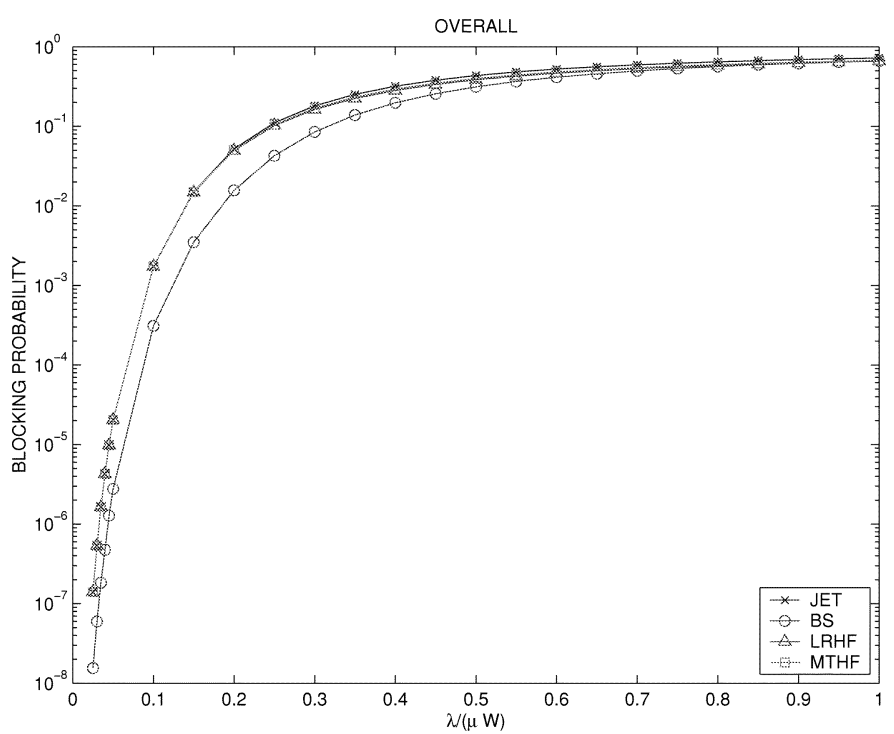

Fig. 9. Overall blocking probabilities (approximation) using JET, BS, LRHF, and MTHF policies.

\section{CONCLUSION}

We have developed a new reduced load fixed-point approximation model to evaluate the blocking probabilities for various implementations of OBS networks. In particular, we have considered JET, BS, and two priority schemes. The accuracy of the approximation was verified by simulations of the NSFNET network.

The numerical results have demonstrated the following phenomena.

1) BS has the lowest overall blocking probability, while the others are similar.

2) Both priority policies improve the overall blocking probability of JET, but not significantly.

3) LRHF is better than MTHF with respect to the overall blocking probability.

4) The LRHF and MTHF priority policies can be used for service differentiation between long and short routes.

\section{REFERENCES}

[1] A. Detti, V. Eramo, and M. Listanti, "Performance evaluation of a new technique for IP support in a WDM optical network: Optical composite burst switching (OCBS)," J. Lightwave Technol., vol. 20, pp. 154-165, Feb. 2002.

[2] K. Dolzer, C. Gauger, J. Spath, and S. Bodamer, "Evaluation of reservation mechanisms for optical burst switching," AEU Int. J. Electron. Commun., vol. 55, no. 1, pp. 18-26, 2001.

[3] D. G. Foursa et al., "2.56 Tb/s $(256 \times 10 \mathrm{~Gb} / \mathrm{s})$ transmission over 11000 km using hybrid Raman/EDFA's with $80 \mathrm{~nm}$ continuous bandwidth," in Proc. OFC 2002, Anaheim, CA, Mar. 2002, pp. FC3 1-FC3 3.

[4] F. P. Kelly, "Blocking probabilities in large circuit-switched networks," Adv. Appl. Probab., vol. 18, pp. 473-505, 1986.

[5] G. M. Louth, "Stochastic Networks: Complexity, Dependence and Routing," Ph.D. Dissertation, Univ. Cambridge, 1990.

[6] J. R. Munkres, Elements of Algebraic Topology. Oxford, U.K.: Perseus Press, 1993, p. 117

[7] C. Qiao, "Labeled optical burst switching for IP-over-WDM integration," IEEE Commun. Mag., pp. 104-114, Sept. 2000.

[8] R. Ramaswami and K. N. Sivarajan, Optical Networks, 2nd ed. San Francisco, CA: Morgan Kaufmann, 2002.

[9] L. Rau et al., "Two-hop all-optical label swapping with varaible length $80 \mathrm{~Gb} / \mathrm{s}$ packets and $10 \mathrm{~Gb} / \mathrm{s}$ labels using nonlinear fiber wavelength converters, unicast/multicast output and a single EAM for 80 - to $10 \mathrm{~Gb} / \mathrm{s}$ packet multiplexing," in Proc. OFC 2002, Anaheim, CA, Mar. 2002, pp. DF2 1-DF2 3.
[10] K. W. Ross, Multiservice Loss Models for Broadband Telecommunication Networks. $\quad$ London, U.K.: Springer-Verlag, 1995.

[11] V. M. Vokkarane, J. P. Jue, and S. Sitaraman, "Burst segmentation: An approach for reducing packet loss in optical burst switched networks," presented at the ICC'02, New York, Apr. 2002.

[12] H. L. Vu and M. Zukerman, "Blocking probability for priority classes in optical burst switching networks," IEEE Commun. Lett., vol. 6, pp. 214-216, May 2002.

[13] D. Wada et al., "Multi-hop 40 Gbits/s variable length photonic packet routing based on multi-wavelength label switching, waveband routing, and label swapping," in Proc. OFC 2002, Anaheim, CA, Mar. 2002, pp. 216-217.

[14] J. Y. Wei, J. L. Pastor, R. S. Ramamurthy, and Y. Tsai, "Just-in-time optical burst switching for multi-wavelength networks," in Proc. 5th Int. Conf. Broadband Commun. (BC'99), 1999, pp. 339-352.

[15] J. Y. Wei and R. I. McFarland, "Just-in-time signaling for WDM optical burst switching networks," J. Lightwave Technology, vol. 18, pp. 2019-2037, Dec. 2000.

[16] J. A. White, R. S. Tucker, and K. Long, "Merit-based scheduling algorithm for optical burst switching," presented at the COIN-PS 2002, Cheju Island, Korea, July 2002.

[17] W. Whitt, "Blocking when service is required from several facilities simultaneously," AT\&T Tech. J., vol. 64, no. 8, pp. 1807-1856, Oct. 1985.

[18] J. Yates, J. Lacey, D. Everitt, and M. Summerfield, "Limited-range wavelength translation in all-optical networks," in Proc. INFOCOM'96, 1996, pp. 954-961.

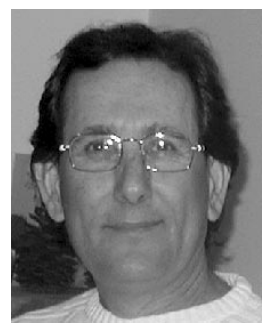

Zvi Rosberg received the B.Sc., M.A., and the Ph.D. degrees from the Hebrew University of Jerusalem, Israel, Jerusalem.

From 1972 to 1978, he was a Senior System Analyst in the Central Computing Bureau of the Israeli Government, where he was one of the chief designers of a new on-line Israeli population registration system. From 1978 to 1979 , he held a Research Fellowship at the Center of Operation Research and Econometric (C.O.R.E.), Catholic University of Louvain, Louvain, Belgium. From 1979 to 1980, he was a Visiting Assistant Professor in the Department of Business Administration, University of Illinois, Urbana, and from 1980 to 1990, he was with the Computer Science Department, Technion-Israel Institute of Technology, Haifa. From 1985 to 1987, he was a Visiting Scientist at IBM Thomas J. Watson Research Center, Yorktown Heights, NY. From 1990 to 1999, he was a Program Manager of communication networks with the Haifa Research Laboratory, Science and Technology, IBM Israel. From 2000 to 2001, he was with Radware Ltd., Tel-Aviv, Israel, as a Chief Scientist. During 2002, he was visiting at the ARC Special Research Center for Ultra-Broadband Information Networks, University of Melbourne, Melbourne, Australia. Currently, he is with the Department of Communication Systems Engineering, Ben Gurion University, Beer-Sheva, Israel. Since 1980, he held summer research positions in IBM Thomas J. Watson Research Center, Department of Electrical and Computer Engineering, University of Massachusetts, Amherst, Department of Electrical Engineering and Computer Science, University of California, Berkeley, and the Radio Communication Systems, Royal Institute of Technology, Stockholm, Sweden. He has published numerous papers in the areas of communication networks, queueing theory, control of queueing systems, applied probability, analysis of algorithms in communication and computing systems, power control and resource allocation in cellular radio systems, and performance evaluation. His current interest and activity is in the area of resource allocation and power control in cellular radio systems, Internet technologies, collaborative computing, and high-speed networking.

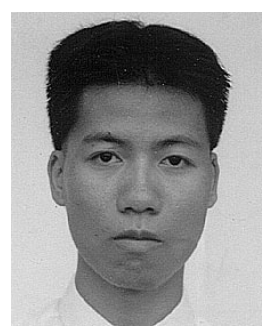

Hai Le Vu (S'97-M'98) received the M.Sc. and $\mathrm{Ph} . \mathrm{D}$. degrees in electrical engineering from the Technical University of Budapest, Budapest, Hungary, in 1994 and 1999, respectively.

From 1994 to 2000, he was a Research engineer at Siemens AG, Hungary. During this period his focus was on performance measurements, Internet quality-of-service (QoS), and IP over ATM. He has been a Research Fellow in the Centre for Ultra-Broadband Information Networks (CUBIN), Department of Electrical Engineering, University of Melbourne, Melbourne, Australia, since 2000. His current research interests are in data network modeling and performance evaluation, optical network design, optimization, applied queuing theory, and the Internet. 


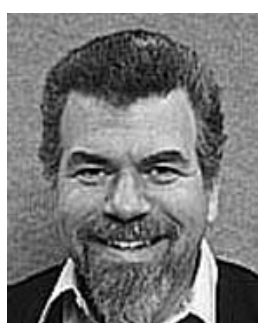

Moshe Zukerman (M'87-SM'91) received the B.Sc. degree in industrial engineering and management, the M.Sc. degree in operation research from Technion-Israel Institute of Technology, and the $\mathrm{Ph} . \mathrm{D}$. degree in electrical engineering from the University of California, Los Angeles (UCLA), in 1985.

He was an independent consultant with IRI Corporation, Los Angeles, CA. He was a Postdoctoral Fellow at UCLA from 1985 to 1986 . From 1986 to 1997, he served in Telstra Research Laboratories (TRL), Clayton, Victoria, Auistralia, first as a Research Engineer and from 1988 to 1997 as a Project Leader. In 1997, he joined The University of Melbourne, Melbourne, Australia, as a Teacher in the Electrical and Electronic Engineering Department and responsible for promoting and expanding telecommunications research. Since 1990, he has also taught and supervised graduate students at Monash University, Melbourne, Australia. He has over 180 publications in scientific journals and conference proceedings and has been awarded several national and international patents.

Dr. Zukerman was the recipient of the Telstra Research Laboratories Outstanding Achievement Award in 1990. He served on the Editorial Board of the Australian Telecommunications Research Journal from 1991 to 1996. He was a Guest Editor of the IEEE JOURNAL ON SELECTED AREAS IN COMMUNICATIONS for two issues: Future Voice Technologies and Analysis and Synthesis of MAC Protocols. Presently, he is serving on the Editorial Boards of the IEEE/ACM TRANSACTIONS ON NETWORKING, the International Journal of Communication Systems, Computer Networks, and as a Wireless Communications Series Editor for the IEEE Communications Magazine. He submitted contributions to and represented Australia in several ITU-T/CCITT standards meetings.

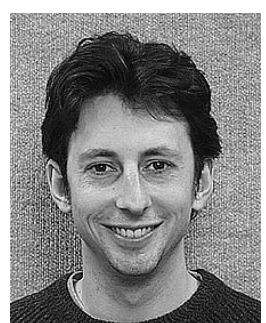

Jolyon White (S'01) received the B.Sc. degree in physics and the B.E. (honors) degree in computer/communications engineering from The University of Melbourne, Melbourne, Australia, in 2001, and is currently working toward the Ph.D. degree at the same university.

During 1997, he took a year off from his studies to work for Innovonics Ltd., Melbourne, Australia, on real-time embedded software for military and industrial transport customers. His research is on design and performance evaluation of optical burst switching and optical packet-switching networks. 